\title{
Comparative Analysis of Various Color Image Compression Techniques
}

\author{
Shreykumar G. Bhavsar \\ PG Research Scholar \\ Department of Electronics \\ and Communication \\ Parul Institute of Engineering \\ \& Technology, Limda, \\ Vadodara, India
}

\author{
Hardik N. Mewada \\ Assistant Professor \\ Department of Electronics \\ and Communication \\ Parul Institute of \\ Engineering \& Technology, \\ Limda, Vadodara, India
}

\author{
Viraj M. Choksi \\ Project Scientist \\ Bhaskaracharya \\ Institute for Space \\ Applications and Geo- \\ Informatics (BISAG), \\ Gandhinagar, India
}

\author{
M. B. Potdar, Ph.D \\ Project Director \\ Bhaskaracharya \\ Institute for Space \\ Applications and Geo- \\ Informatics (BISAG), \\ Gandhinagar, India
}

\begin{abstract}
Recent research has focused on the image compression algorithms are lossy compression. However, the general algorithm for certain types of images, resulting in poor image quality is usually implemented using a baseline quantization process, especially in the combination of image content. It's a lot different compression image coding based on Wavelet transform technology utilizes signal. A different type of variable image compression algorithms like JPEG, JPEG2000, EZW, SPIHT compression technology which can be used is very important.
\end{abstract}

\section{General Terms}

Compression, Color images.

\section{Keywords}

Discrete Wavelet Transform (DWT), JPEG, JPEG2000, EZW, SPIHT, Peak signal to Noise Ratio (PSNR), Mean Square Error (MSE), Compression Ratio (CR).

\section{INTRODUCTION}

The presence of large amounts of data in a form of digital image data. Currently, expand the image size and resolution requirements. The best result of the expansion is less distinct image icon in the Tools acquits. Digital imaging technology can only be restored, because the firm's conduct [11]. In the literature, a number of algorithms were introduced. High compression ratio for low bit of the wavelet transform coefficients to form a zero or very near zero. Image compression without reducing image quality, number of bytes in the image file size is reduced. The size of the image file to a predetermined amount of disk or memory space to store the majority of resolutions are permitted. It also reduced the time needed to download from the internet to an image or web page.

Compression is a method to produce a compact representation of the digital information signal. In the literature, the terms source coding, data compression, bandwidth compression, and compression are all used to refer to process of image compression [14]. If the signal is defined as an image, a video stream or an audio signal, the general problem is to minimize the compressed bit rate of the digital representation. There beneficial when images, video and audio signals may be compressed in the form of many applications. Uncompressed, most of these applications are not feasible.

\section{IMAGE COMPRESSION PROCESS}

Compression and decompression of the basic block diagram in Fig 1 and Fig 2. Compression process involves the use of discrete wavelet transform of the input image, and the coded coefficients quantized wavelet coefficients. Application of vacuum or compressed decoding and inverse bit stream to the process of the wavelet transform factor involved in the reconstructed image [10]. Next Steps quantization error introduced has named for lossy compression. Calculating a threshold value is a lossy compression technology is the most important part in this processing. It contains for the high threshold value, the higher the compression ratio but the poor image quality and vice versa [13].

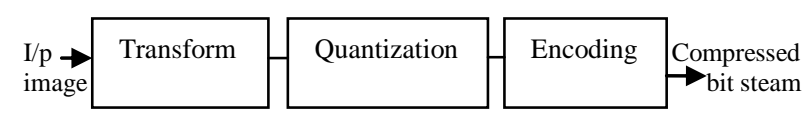

Fig 1: Image Compression process [2]

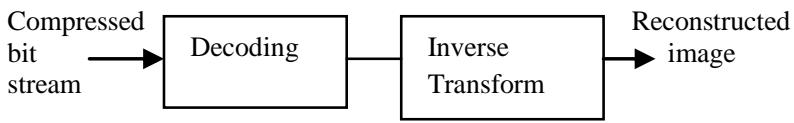

Fig 2: Decompression Process [2]

\section{DISCRETE WAVELET TRANSFORM}

In general, we use wavelet transform to check the activity signal that signal, the frequency response changes over time Fourier transform is not suitable for such a signal. Wavelet transform includes the original signal on the basis of each of its elements is called the mother wavelet function and which change the mixed of the report of the coefficients [11]. The image processing for pattern recognition problems are difficult to solve and computer graphics. Wavelet analysis is a new exciting approach. Wavelet makes complex information such as images, patterns in different locations and scale decomposition as the basic form and then rebuilds the high accuracy. Discrete wavelet transform of a signal is passing to it through a series of filters calculations. Both through a low pass filter impulse response convolution result of the first sample. The decomposed signal is also simultaneously a high pass filter. For Wavelet decomposition one level at output enable (from the high-pass filter) detail coefficients and approximation coefficients (low-pass) [4]. It is important that both filters are related to each other. They are called quadrature mirror filters. Sometime half of the frequency has not used. That's way half the Nyquist sample may be discarded according to the rules. The filter output has been sub-sampled and this decomposition of time resolution has reduced by half because each filter output has carried only half of the signal characteristics. So that each output having 
half of the input frequency and then frequency resolution is doubled [4].

\begin{tabular}{|c|c|c|c|}
\hline LL3 & HL3 & & \\
LH3 & HH3 & HL2 & HL1 \\
LH2 & HH2 & \\
\hline & & \\
& & \\
& & \\
& & \\
& & \\
& & \\
& & \\
& & \\
\end{tabular}

Fig 3: Pyramidal structure of 3-level wavelet decomposition [12]

For image applications for separable two dimensional discrete wavelet transform is normally performed by which each row of the image is first transformed using the bank of filter. The same bank of filter is then applied vertically to each column of the transformed image [12]. The result is four sub bands termed LL1 (horizontally and vertically low-pass), HL1 (horizontally high-pass and vertically low-pass), LH1 (horizontally low-pass and vertically high-pass) and HH1 (horizontally and vertically high-pass). To increase the efficiency of the Wavelet Transform for various decomposition stages is recursively performed on the LL1 sub band because it is smoothed version of the original image and thus still highly correlated i.e.LL1 is decomposed to LL2, HL2, LH2, and HH2 and so on [12].

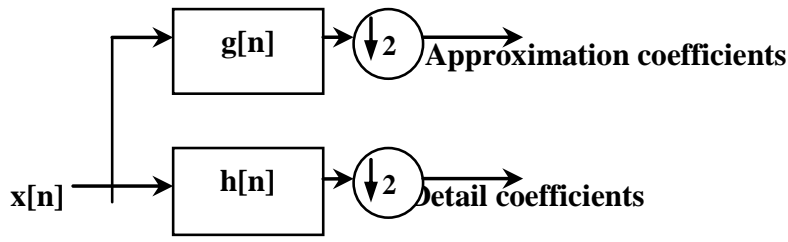

Fig 4: Wavelet Decomposition one level [4]

Repeat decomposition to further improve the frequency resolution, a high and low pass filter approximation coefficients and the decomposition of the sample [4]. This is shown in figure as having said different time-frequency localization subspace node binary tree and these trees are called filters. The following statistics of the level of each signal is decomposed into low and high frequencies. Due to decomposition level the input signal must be a multiple of $2^{\mathrm{n}}$, where $n$ is number of levels [4].

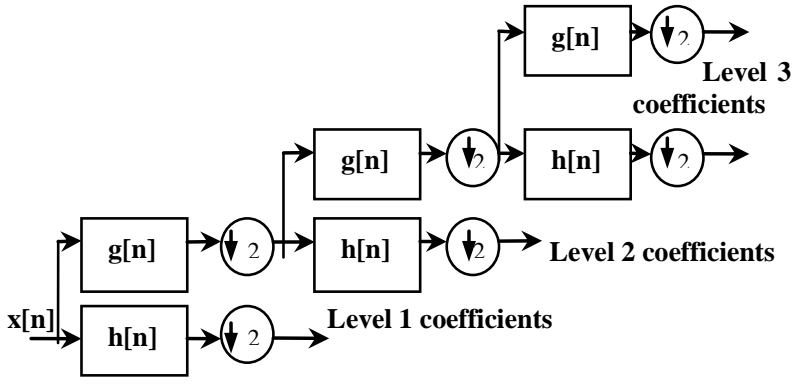

Fig 5: Wavelet Decomposition two levels [4]

\section{COLOR IMAGE COMPRESSION}

A color image compression is very important in today's era of communication because most of the image is in color form. Color image is required more space for storage. Without compression, it may take a more time to transfer color images via the internet or anywhere [1]. Fig 6 shows a model for the color image compression.

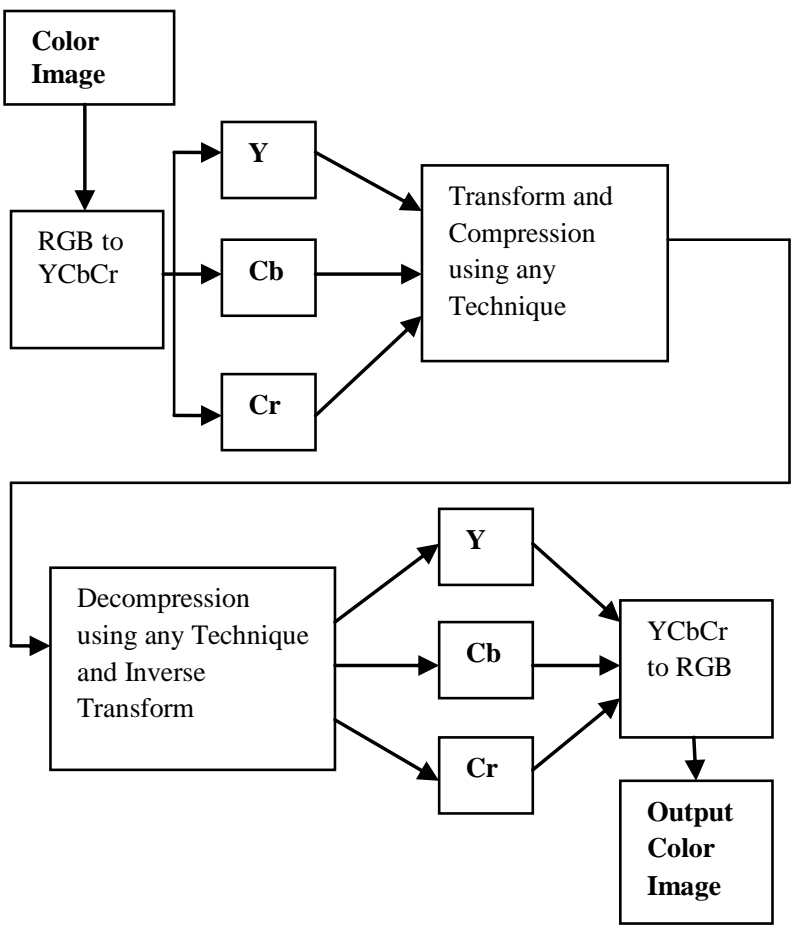

Fig 6: Method of color image compression [1]

Color image (RGB-24 bits) is converted to $\mathrm{YCbCr}$ format. $\mathrm{YCbCr}$ or $\mathrm{Y}^{\prime} \mathrm{CbCr}$ sometimes written or $\mathrm{YCBCR} \mathrm{Y}^{\prime} \mathrm{CBCR}$, the color space is used as part of a digital video camera system and a color image of a family pipeline [1]. $\mathrm{Y}^{\prime}$ is the luminance component, $\mathrm{Cb}$ and $\mathrm{Cr}$ are the difference in blue, red color difference chrominance components. $\mathrm{Y}^{\prime}$ (with primes) from $Y$, which is the luminance difference, this means that the intensity of light using a non-linear gamma correction encoding [1]. Wavelet analysis is converted to $\mathrm{Y}, \mathrm{CB}$ and CR.

$\mathrm{Y}$, which is the luminance and it represents the brightness of light which is proportional to the total energy in the visible light band, $\mathrm{Cb}$, which is the difference between the gray color and blue color, $\mathrm{Cr}$, which is the difference between the gray color and red color. 


\section{COMPRESSION TECHNIQUES}

Over the years a variety of linear transforms have been developed which include Discrete Fourier Transform (DFT), Discrete Cosine Transform (DCT), Discrete Wavelet Transform (DWT) and many more other transform but each with its own advantages and disadvantages.

Many discrete cosines transform conversion and the input is converted to a linear combination of weighted basis functions. These basis functions are mainly used frequencies, such as a sine wave. A two-dimensional DCT cosine is different, the $\mathrm{x}$ and y direction is applied to transform one another. Recently, wavelet transform has become a cutting-edge technology of image compression research work. As can be seen it provides a significant improvement in image quality at higher compression ratio, mainly due to better energy wavelet compression DWT based on wavelet transform coding. After a few years, a variety of powerful and sophisticated image compression scheme based on wavelet transform functions have been developed and implemented. There are many benefits and the new standard is based on the top contenders in the wavelet compression algorithm.

\subsection{JPEG}

JPEG (Joint Photographic Experts Group) standard, for a time and only criterion is that the lossy compression of still images. There are interesting in the JPEG standard technique quite useless. JPEG from the introduction of this standard names committee. Image compression is a variable type. This is the effective use of the static image [14].

JPEG is lossy image reconstructed at the receiver end but not the same as transmitter side. Advantageously, it can be achieved a much higher compression with this standard but at the cost of some of the data. The slight difference in color is not visible to the human eye and works on the concept of the JPEG standard. If completed a detailed analysis of the image pixel by pixel, it will be appreciated that the amount of data loss is high. Although the data is lost, it can be controlled by setting parameters [15]

A reduced file size can be maintained to reduce the number of bits, until it does not affect image quality. At the receiving end should be good image recognition. There is a mode called progressive scan mode, it can handle real-time image transmission. [14]. JPEG image compression is based on the following main steps: The image is separated into three color components. Each component does not overlap is divided into $8 \times 8$ block. Each block is transformed using the twodimensional Discrete Cosine Transform (DCT). Each block of quantized transform with respect to a quantization matrix of $8 \times 8$, it may be independently selected for all three color channels. The resulting data is compressed using Huffman or arithmetic coding [15].

\section{Advantages [16]:}

- JPEG algorithm is almost universal. The technology has low complexity. This is partly due to the fact the case, it has been around for a long time.

- JPEG can handle a variety of color and keep the file size to a minimum and its memory efficiency.

- JPEG file compression, which means that a JPEG image will be captured photos smaller than other formats and this makes it easier to store JPEG files and e-mail.
- JPEG format has a more reasonable coding efficiency. JPEG images are stored by the fast cameras and other devices. This enables users to capture fast moving action and JPEG image will become blurred in a higher resolution image.

- This allows the JPEG image in the default file format used on the internet and the image can be compressed to up to $5 \%$ of its original size with the transmission of information to the web pages.

- This algorithm can be saved in a sliding scale based on the desired resolution quality.

\section{Disadvantages [16]:}

- JPEG provides an excellent quality in the high and medium bit rate. However, the quality is unacceptably low bit-rate compression.

- In lossless and lossy compression in a single stream JPEG can provide excellent performance.

- The current JPEG standard provides a number of coincident indicators but the quality is still lower bit error is encountered and it has a transmission in a noisy environment.

- JPEG images are optimized as a natural. Its poor computer generated images and binary image performance.

- Image compression method allows the user to save the file in a relatively small but the cost in image quality and detail.

- JPEG images are lower quality than other techniques and especially at low resolutions.

- Lossless JPEG mode in an attempt to moderate the loss of information in the JPEG compression.

\subsection{JPEG2000}

JPEG2000 standard for provides a set of features that are important to many high end and appearing applications to take advantage of new technologies [5]. Some of the most important features that standard criteria are the following:

- This algorithm has a improve compression efficiency.

- Jpeg2000 have to utilize for lossy to lossless compression purpose.

- It has to multiple resolution representation and also improves the image quality.

- This method has progressive transmission by pixel accuracy and resolution.

- It has to more interesting on region of interest coding

- This algorithm has random access processing and also continuous bi-level compression.

JPEG2000 is the quality scalability through the use of ratedistortion optimization technique is formed by the encoder, the layer quality incorporated [5]. Quality scalability is an important feature by which allows several bit rates and without sacrificing performance coding as well as decoding of the transmission or compressed code stream [2]. JPEG2000 is inherently more complex than its predecessor JPEG format. Its reliance on discrete wavelet transform (DWT) and the coding block of wavelet coefficients and also means a 
significantly higher cost in memory consumption than the baseline JPEG [2].

JPEG2000 standard process consists of five steps: image segmentation and code block, quantization, embedded block coding with optimal truncation (EBCOT) first layer coding, best truncated and EBCOT II line coding [5]. Wavelet image is converted to a different types of standard as DWT is used to transform. Destructive processing steps reduces the compression performance of the entropy of the original image and the image transformation in order to help out code block segmentation to separate them into separate components and also the encoded block data. Lossy quantization process, thus it has reducing the dynamic range of the transformed image components, reducing the size and quality of the reconstructed output image file. In many line coding process requires that's processing and lossless compression code block independently. Best truncation process is disadvantageous process for each of which further reduces the size of a block of compressed codes until the desired file size to achieve. After the optimal cut off treatment, respectively by EBCOT layer II encoder encodes the block and ordered its JPEG2000 file format [5].

\section{Advantages [2][5]:}

- Mainly the algorithm has better compression quality because based on the use of wavelet transform this approach.

- $\quad$ Progressive is achieved by passing the resolution wavelet decomposition organized stream and progress is determined by the quality and also color coded bit-plane is a progressive organization that has achieved through the color channels in the file implementation.

- The standard is very flexible to allowing a huge component subsamples image and different bit depth of each channel and other changes.

- This allows the encoder to select a higher quality than the rest of the image to be encoded in a region of the image.

- This is achieved by a packet stream to be achieved. Some optional features such as resynchronize mark and also the table of contents packet marking and add more anti start packet error mark ends.

\section{Disadvantages [2][5]:}

- This is mainly used for the appropriate program or plugin to watch.

- This method has been used for more demanding in memory.

- This technique has a higher consumption of computing time.

- This technology is not widely used in real life.

\subsection{EZW algorithm}

The EZW encoder was originally designed to operate on an image (two-dimensional signal), but it can also be used in other dimensional signals. It is based on the progressive coding to the compressed image bit stream in order to improve accuracy [6]. This means that when more bits are added to the data stream that time the decoded image will contain more detail and similar to the properties of JPEG coded images. Using the embedded coding algorithm, the encoder or the target speed may be sufficiently accurate to meet the target at any time and in the presence of the termination code [7]

Features of EZW algorithm [3]:

- It has significant wavelet coefficients provide a compact binary zero trees coding mapping. There successive approximation wavelet coefficients quantify but it includes a multi-level adaptive arithmetic coding.

The EZW algorithm is based on four key concepts [3]:

1. This process must use discrete wavelet transform.

2. A significant lack of information through the use of selfsimilarity across prediction inherent scaling images.

3. In this algorithm has been required to quantify entropy coding successive approximation.

4. This is accomplished by a "Generic" adaptive arithmetic coding to achieve lossless data compression.

Advantages [6]:

- $\quad$ EZW compression of the core is different scales of selfsimilarity of the entire image using wavelet transform.

- EZW algorithm is considered to be the first truly effective wavelet encoder.

- Adaptive arithmetic coder is used to obtain a higher entropy compression.

- EZW approximate frequency coefficients of wavelet transform of the image. Because the wavelet transform coefficients and spatial frequency content that contains information about the image for discarding the high frequency coefficients of the image will result in the recovery of some image degradation specific position rather than the entire image.

\section{Disadvantages [6]:}

- The algorithm scans the sub-band wavelet coefficients in a zigzag manner by a sub-band.

- The main feature of the method of coded data can be transmitted explicitly order.

- An important fact used in the sorting algorithm design is that we do not need all the coefficients sorted.

\subsection{SPIHT algorithm}

SPIHT coding attributes powerful embedded information sorted needs and requirements and also to reduce the error correcting code from the beginning to the end of the compressed file [9]. If an error is detected but not corrected, the decoder point still refused to receive and display digital images obtained before the data and error. It generates two types of data, the first type of information needs to be protected and using the second error does not require special protection because they affect only one pixel addition between symbols and bit uncompressed [3]. Group pixels resolution wavelet tree originated from the lowest bandwidth. Coefficients are divided into $2 \times 2$ array and the difference is a factor of the frequency band which is a descendant coefficient lower resolution band. Again the minimum coefficient of the strip is divided into $2 \times 2$ array [12].

SPIHT algorithm based on the concept [9]: 
- In this algorithm must use the ordered planes progressive transmission. Must aim to set the partition sort sorting algorithm and using spatial orientation tree.

Advantages [3]:

- It can be interrupted at any time and the decoded result to the maximum possible detail one bit accuracy can be reused.

- This is a small portion of the user transmitted from a slower connection speeds can download the file and relative to other codes such as progressive JPEG is also used to obtain the results over the network is useful documents.

- It is very compact bigger change output bit stream. No additional entropy coding has been applied.

Disadvantages [3]:

- It is susceptible to corruption as a single-bit error bit can introduce significant distortion of the image depending on its position.

- It is the bit synchronization attributes because leakage can result in the transmission bit is completely distorted from the side of the decoder.

\section{EXPERIMENTAL PARAMETERS}

\subsection{PSNR comparison}

Computational formula of Peak Signal to Noise Ratio (PSNR) and mean square error (MSE).

$$
\begin{aligned}
& \text { PSNR }=10 * \log \left[\frac{\left(2^{\mathrm{n}}-1\right)^{2}}{\mathrm{MSE}}\right] \\
& M S E=\sum_{\mathrm{i}=0}^{\mathrm{K}-1} \sum_{\mathrm{j}=0}^{\mathrm{M}-1}\left[\frac{(\mathrm{f}(\mathrm{i}, \mathrm{j})-\mathrm{g}(\mathrm{i}, \mathrm{j}))^{2}}{\mathrm{M} * \mathrm{~K}}\right]
\end{aligned}
$$

Where, $f(i, j)=$ original image, $g(i, j)=$ reconstructed image, $n$ bits per pixel

\subsection{Comparison of different methods and Literature Survey}

\begin{tabular}{|c|c|c|c|c|}
\hline $\begin{array}{c}\text { Image } \\
\text { Compression } \\
\text { Techniques }\end{array}$ & $\boldsymbol{C R}$ & $\boldsymbol{M S E}$ & $\boldsymbol{P S N R}$ & $\begin{array}{c}\text { Memory } \\
\text { Utilization }\end{array}$ \\
\hline JPEG & Low & High & Low & High \\
\hline JPEG2000 & High & High & Low & High \\
\hline EZW & High & High & Low & Low \\
\hline SPIHT & High & Low & High & Low \\
\hline
\end{tabular}

\subsection{PSNR values for different image compression techniques [3][6][10][11]}

\begin{tabular}{|c|c|c|}
\hline Image Compression Techniques & Bit rate & PSNR \\
\hline JPEG & 0.5 & 26.74 \\
\hline JPEG2000 & 0.5 & 27.98 \\
\hline EZW & 0.5 & 29.75 \\
\hline SPIHT & 0.5 & 30.23 \\
\hline
\end{tabular}

\section{CONCLUSION}

This paper is focused on the comparison among four important techniques of color image compression. Various Image compression techniques for an image is done based on three parameters Compression Ratio (CR), Mean Square Error (MSE) and Peak Signal to Noise Ratio (PSNR) and also introduced the JPEG, JPEG2000, EZW, SPIHT algorithms of color image compression. Among these all different image compression, concluded that SPIHT algorithm is good quality image, high compression ratio, less mean square error value, less memory utilization and higher PSNR at higher bpp for different types of color images.

\section{ACKNOWLEDGMENTS}

For the successful completion of this review article, we thank all scholars, guides, family members and dear friends who directly or indirectly contribute to making our job done.

The Authors wish to thank T.P.Singh, Director, BISAG for constant encouragement to our research work.

\section{REFERENCES}

[1] Sadashivappa, K.V.S Anand Babu and Dr. Srinivas K, "Color Image Compression using SPIHT Algorithm", International Journal of Computer Applications(IJCA), Vol. 16, No.7, February 2011.

[2] Charilaos Christopoulos, Touradj Ebrahimi, Athanassios Skodras, "The Jpeg2000 Still Image Coding System: An Overview", IEEE Transactions on Consumer Electronics, Vol. 46, No. 4, pp. 1103-1127, November 2000.

[3] Sure. Srikanth, Sukadev Meher, "Compression Efficiency for Combining Different Embedded Image Compression Techniques with Huffman Encoding", International conference on Communication and Signal Processing, April 3-5, 2013.

[4] Vidhi Dubey, Rahul Dubey, "A new Set Partitioning in Hierarchical (SPIHT) Algorithm and Analysis with Wavelet Filters" International Journal of Innovative Technology and Exploring Engineering (IJITEE), August 2013.

[5] Reena Singh, V.K.Srivastava, "JPEG2000: A review and its performance comparison with JPEG", International Conference on Power, Control and Embedded Systems (ICPCES), 2012.

[6] V.S.Shingate, S.N.Talbar and T.R. Sotakke, "Still Image Compression using Embedded Zero tree Wavelet Encoding", International Journal of Computer Science \& Communication, Vol.1, pp.21-24, June 2010,.

[7] C.D., Creusere, "A New method of Robust Image Compression Based on the Embedded Zero tree Wavelet 
Algorithm", IEEE Transactions on Image processing, No. 10, pp.1436-1442, 1997.

[8] Tripatjot Singh, Sanjeev Chopra, Harmanpreet Kaur, Amandeep Kaur, "Image compression Using Wavelet and Wavelet packet Transformation" IJCST Vol.1, Issue 1, September 2010.

[9] A.Said and W.A.Pearlman, "A new fast and efficient image codec based on set partitioning in hierarchical trees" IEEE Transactions on Circuits and systems for Video Technology, vol.6, no.3,pp.243-250,Jun.1996.

[10] N.B. Chopade, A. A. Ghatol and M. T. Kolte, "Efficient Image Compression and Transmission Using SPECK", SPIT-IEEE Colloquium and International Conference, Mumbai, India, 2005.

[11] Puja D Saraf, Deepti Sisodia, Amit Sinhal and Shiv Sahu, "Design and Implementation of Novel SPIHT Algorithm for Image Compression", IEEE International Conference on Parallel, Distributed and Grid Computing, 2012.
[12] Ali Kadhim, Al-Janabi, "Low Memory Set-Partitioning in Hierarchical Trees Image Compression Algorithm", International Journal of Video \& Image Processing and Network Security (IJVIPNS-IJENS), Vol.13 No.02, 2013.

[13] K Sayood, "Introduction to Data Compression", Morgan Kaufm ann, San Fransisco, USA, 1996.

[14] http://en.wikipedia.org/wiki/JPEG

[15] Heinz H. Bauschke, Christopher H. Hamilton, Mason S. Macklem, Justin S. McMichael and Nicholas R. Swart, "Recompression of JPEG Images by Requantization", IEEE Transactions On Image Processing, Vol. 12, No. 7 July 2003.

[16] http://www.zicara.com/techbytes/webdesign/advantagesdisadvantages-jpeg 